\title{
Do antiepileptics have any potential to induce insulin resistance? A comparison between levetiracetam and valproic acid
}

\author{
Gökhan Özer® \\ Department of Neurology, Sanko University School of Medicine, Gaziantep, Turkey
}

DOI: $10.18621 /$ eurj.382538

\begin{abstract}
Objective: To explore whether levetiracetam and valproic acid are associated with insulin resistance and to determine their effects on glucose and insulin levels among different age groups.

Methods: Demographic data were collected for epileptic patients from different age groups with a normal body mass index and no chronic endocrine disease who were on levetiracetam or valproic acid therapy. In addition to routine laboratory tests, fasting insulin level was checked and Homeostatic Model Assessment for insulin resistance calculated for all patients. Based on these measurements, the effects of levetiracetam and valproic acid on laboratory parameters were compared statistically.

Results: A total of 61 patients (30 males, 31 females) diagnosed with epilepsy were included in the study. Thirty-four (55.7\%) the patients were less than 15 years of age and the others were older. Twenty-three (37.7\%) patients were on valproic acid therapy and $38(62.3 \%)$ patients were receiving levetiracetam. Thirty-two $(52.5 \%)$ the patients tested positive for insulin resistance and others were insulin resistance-negative. Nine $(28.1 \%)$ patients on valproic acid treatment and $71.9 \%(n=23)$ of patients on levetiracetam treatment were insulin resistance-positive.

Conclusions: In the present study, contrary to the published literature, levetiracetam was found to be associated with further negative effects on insulin and blood glucose metabolism compared to valproic acid. We determined that levetiracetam had a distinct pharmacokinetic profile in pediatric patients as demonstrated by its effects on glucose and insulin metabolism.
\end{abstract}

Keywords: insulin resistance, levetiracetam, epilepsy, valproic asid

Received: January 23, 2018; Accepted: February 25, 2018; Published Online: April 10, 2018

A 1 ltered patterns of hormonal secretion due to direct action of epileptic discharges have been shown in humans and animals, but it is difficult to establish the underlying cause of untoward effects of antiepileptic medications which can be multifactorial in epileptic patients. Monotherapy should be preferred whenever possible to avoid occurrence of adverse effects and complicated problems [1-5].

However, prolonged use of antileptic drugs is known to be associated with adverse effects such as metabolic and organ toxicity, endocrine disturbance, negative cognitive effects, and psychiatric problems, particularly with alterations in thyroid function in patients withepilepsy. Some antileptic drugs are

Address for correspondence: Gökhan Özer, MD., Assistant Professor, Sanko University School of Medicine, Department of Neurology, Gaziantep, Turkey

E-mail:primernordr@gmail.com 
enzymes responsible for vitamin $\mathrm{D}$ metabolism to the inactive metabolites of $25(\mathrm{OH}) 2 \mathrm{D}$ vitamin. 1.25 $(\mathrm{OH}) 2 \mathrm{D}$ vitamine level Decreases the calcium absorption so the second hyperparathyroidism, increased bone resorption and bone loss it is developing [6-9].

Studies have clearly demonstrated the impact of antiepileptic drugs, on hormonal status including effects on fertility, sexual function, bone structure and thyroid hormone. While studies evaluating thyroid function status in epileptic patients suggest that thyroid hormone levels are not directly related to epilepsy, some changes in thyroid function tests could be associated with the prescribed antileptic drugs [10].

Levetiracetam is structurally unrelated to other antiepileptic drugs although its effect on CYP450 enzyme induction is not clear. Levetiracetam specifically binds to the synaptic vesicle protein (SV2A) which is commonly found in the central nervous system and endocrine tissues [11]. It was observed that levetiracetam induces secretion of testosterone and estrogen without stimulating release of gonadotropin from ovarian follicular cells. This observation suggests that endocrine functions are affected by levetiracetam particularly in women of childbearing age [12]. Previous studies have shown that thyroid functions are not significantly influenced by LEV in children and adults [13]. In case-control studies, valproic acid was shown to lower blood glucose concentration independent of weight gain and hyperinsulinemia [14]. There are no studies in literature on the impact of levetiracetam on serum insulin and/or glucose levels.

\section{METHODS}

Non-diabetic patients with epilepsy for at least one year, using epilepsy medication for at least a year and a normal body mass index who admitted to Gaziantep Sanko University School of Medicine in 2017 and were on monotherapy were included in the study after being examined by a neurologist. Ethical approval was obtained from the ethics committee of the same hospital. Patients were stratified into two groups using 15 years of age as a cut-off (Group 1, less than $15 \mathrm{y}$ and Group 2, $\geq 15$ y). Patients receiving levetiracetam were compared with those on valproic acid. Valproic acid was chosen for the study due to its potential to induce weight gain and hyperinsulinemia and known effects on endocrine metabolism. In addition to routine biochemistry panel, fasting insulin values were measured for all patients. Medical history, age, and gender data were recorded by specialist neurologist. Height and body weight were measured to obtain body mass index $(\mathrm{kg} / \mathrm{m} 2)$ and age- and sex-specific body mass index percentiles. Associations between average doses of study medications and blood glucose, insulin and HOMA-IR values were examined in relation to age and gender. Insulin resistance was calculated using the The homeostasis model assessment of insulin resistance (HOMA-IR) formula of fasting insulin $(\mathrm{uU} / \mathrm{mL}) \times$ fasting glucose $(\mathrm{mmol} / \mathrm{L}) / 405$ using a cutoff value of 2.5 [15]. HOMA-IR is a noninvasive and effective alternative method to evaluate insulin sensitivity based on the glucose level and the level of serum insulin measured in fasting conditions. HOMAIR is considered a standard method of measuring insulin resistance in epidemiological studies [16].

\section{Statistical Analysis}

Normality assumption for numerical variables was checked using the Shapiro-Wilk normality test. MannWhitney $U$ test was used to compare numerical variables between independent groups and chi-square test was applied to investigate the relationship between categorical variables. Statistical analyses were conducted in SPSS for Windows, version 24.0 and a $p$ value less than 0.05 was considered statistically significant.

Table 1. Demographic characteristics of patients

\begin{tabular}{llcc}
\hline Variables & & $\mathbf{n}$ & $\mathbf{\%}$ \\
\hline Age (years) & $<15$ & 34 & 55.7 \\
\multirow{3}{*}{ Gender } & $\geq 15$ & 27 & 44.3 \\
& Male & 30 & 49.2 \\
Medication & Female & 31 & 50.8 \\
& VPA & 23 & 37.7 \\
HOMA-IR status & LEV & 38 & 62.3 \\
& IR (+) & 32 & 52.5 \\
& IR (-) & 29 & 47.5 \\
\hline
\end{tabular}

HOMA-IR $=$ Homeostatic model assessment for insulin resistance, IR $(-)=$ insulin resistance-negative, IR $(+)=$ insulin resistance-positive, $\mathrm{LEV}=$ levetiracetam, $\mathrm{n}=$ the number of patients, VPA = valproic acid 


\section{RESULTS}

A total of 61 patients were included in the study, of whom $30(49.2 \%)$ were males. Thirty-four patients were less than 15 years of age and the others were older. Twenty-three (37.7\%) patients were on valproic acid therapy and the remaining patients were on levetiracetam therapy. Thirty-two $(52.5 \%)$ patients were tested positive for insulin resistance and other patients were insulin resistance-negative (Table 1).
Mean glucose value was $92.2 \mathrm{mg} / \mathrm{dL}$, mean insulin value was $26.2 \mu \mathrm{U} / \mathrm{mL}$ and mean HOMA-IR value was 5.94 among insulin resistance-positive patients receiving valproic acid (Table 2). Mean glucose value was $95.91 \mathrm{mg} / \mathrm{dL}$, mean insulin value was $21.2 \mu \mathrm{U} / \mathrm{mL}$ and mean HOMA-IR value was 5.29 among insulin resistance-positive patients receiving levetiracetam (Table 3). Among patients less than 15 years of age, $46.9 \%$ insulin resistance-positive and in patients older than 15 years of age, $53.1 \%$ were insulin resistance-

Table 2. Relationship between valproic acid, glucose, insülin, HOMA-IR

\begin{tabular}{lccc}
\hline VPA group & $\begin{array}{c}\text { IR }(+) \\
(\mathbf{n}=\mathbf{9})\end{array}$ & $\begin{array}{c}\text { IR }(-) \\
(\mathbf{n}=\mathbf{1 4})\end{array}$ & $\boldsymbol{p}$ value \\
\hline Glucose $(\mathbf{m g} / \mathbf{d L})$ & $92.22 \pm 16.79$ & $83.57 \pm 9.43$ & 0.305 \\
Insulin $(\boldsymbol{\mu U} / \mathbf{m L})$ & $26.26 \pm 12.67$ & $35.72 \pm 116.39$ & $\mathbf{0 . 0 0 1}$ \\
HOMA-IR & $5.94 \pm 3.02$ & $0.94 \pm 0.65$ & $\mathbf{0 . 0 0 1}$ \\
\hline
\end{tabular}

HOMA-IR = Homeostatic model assessment for insulin resistance, IR (-) = insulin resistancenegative, IR $(+)=$ insulin resistance-positive, $\mathrm{n}=$ the number of patients, VPA $=$ valproic acid

Table 3. Relationship between levetiracetam, glucose, insülin, HOMA-IR

\begin{tabular}{lccc}
\hline LEV group & IR $(+)$ & IR $(-)$ & $p$ value \\
& $(\mathbf{n}=\mathbf{2 3})$ & $(\mathbf{n}=\mathbf{1 5})$ & \\
\hline Glucose $(\mathbf{m g} / \mathbf{d L})$ & $95.91 \pm 8.18$ & $81.67 \pm 24.42$ & $\mathbf{0 . 0 0 6}$ \\
Insulin $(\boldsymbol{\mu U} / \mathbf{m L})$ & $21.8 \pm 13.29$ & $75.47 \pm 173.69$ & $\mathbf{0 . 0 0 5}$ \\
HOMA-IR & $5.29 \pm 3.55$ & $1.59 \pm 1.61$ & $\mathbf{0 . 0 0 1}$ \\
\hline
\end{tabular}

HOMA-IR = Homeostatic model assessment for insulin resistance, IR (-) = insulin resistancenegative, $\mathrm{IR}(+)=$ insulin resistance-positive, $\mathrm{LEV}=$ levetiracetam, $\mathrm{n}=$ the number of patients

Table 4. Relationship between age, gender, medication and insulin resistance.

\begin{tabular}{lcccccc}
\hline Variables & & \multicolumn{2}{c}{ IR (+) } & \multicolumn{2}{c}{ IR (-) } & p value* \\
& & n & \% & n & \% & \\
\hline Age (years) & $<15$ & 15 & 46.9 & 19 & 65.5 & 0.143 \\
\multirow{3}{*}{ Gender } & $\geq 15$ & 17 & 53.1 & 10 & 34.5 & \\
& Male & 14 & 43.8 & 16 & 55.2 & 0.373 \\
Medication & Female & 18 & 56.2 & 13 & 44.8 & \\
& VPA & 9 & 28.1 & 14 & 48.3 & 0.105 \\
& LEV & 23 & 71.9 & 15 & 51.7 & \\
\hline
\end{tabular}

IR $(-)=$ insulin resistance-negative, IR $(+)=$ insulin resistance-positive, $L E V=$ levetiracetam, $\mathrm{n}$ $=$ the number of patients, VPA $=$ valproic acid, $*$ Chi-square test

Table 5. Comparison of total dose between IR (+) and IR (-) groups.

\begin{tabular}{lcccccc}
\hline Variable & Medication & $\mathbf{n}$ & IR (+) & $\mathbf{n}$ & IR (-) & p value \\
\hline Total dose & VPA & 9 & $1922.22 \pm 3068.7$ & 14 & $489.29 \pm 203.98$ & $\mathbf{0 . 0 1 6}^{*}$ \\
(mg/day) & LEV & 23 & $860.87 \pm 501.58$ & 15 & $860 \pm 636.73$ & 0.768 \\
& Overall & 32 & $1686.35 \pm 507.52$ & 29 & $681.03 \pm 507.52$ & $\mathbf{0 . 0 4 6}^{*}$ \\
\hline
\end{tabular}

IR (-) = insulin resistance-negative, IR $(+)=$ insulin resistance-positive, LEV $=$ levetiracetam, $n$

$=$ the number of patients, VPA $=$ valproic acid, *Mann-Whitney U test 
Table 6. Relationship between medication use and IR for different age groups

\begin{tabular}{lcccccc}
\hline Age (years) & Medication & \multicolumn{2}{c}{ IR (+) } & \multicolumn{2}{c}{ IR (-) } & \multirow{2}{*}{ p value* } \\
& & n & \% & n & \% & \\
\hline$<\mathbf{1 5}$ & VPA & 5 & 33.3 & 12 & 63.2 & 0.084 \\
& LEV & 10 & 66.7 & 7 & 36.8 & \\
\multirow{2}{*}{$\mathbf{1 5}$} & VPA & 4 & 23.5 & 2 & 20.0 & 0.831 \\
& LEV & 13 & 76.5 & 8 & 80.0 & \\
\hline
\end{tabular}

IR (-) = insulin resistance-negative, IR (+) = insulin resistance-positive, LEV = levetiracetam, $\mathrm{n}=$ the number of patients, VPA $=$ valproic acid, ${ }^{*}$ Chi-square test

positive. Insulin resistance was detected in $56.2 \%$ of female patients. Twenty-three (71.9\%) patients receiving levetiracetam treatment and $28.1 \%(\mathrm{n}=9)$ of patients receiving valproic acid treatment were insulin resistance-positive. No significant association was found between insulin resistance and pharmacological agents in question, age, and gender when analyzed by chi-square test (Table 4 ). Total dose was significantly higher in insulin resistance-positive patients for valproic acid $(p=0.016)$ and overall comparison $(p=0.046)$. However, no significant difference was detected for levetiracetam $(p=0.768)$ (Table 5). There was no statistically significant association between valproic acid or levetiracetam use and insulin resistance in different age groups (Table 6). Insulin resistance was more likely to develop with increased dose of medication, particularly among patients using valproic acid (Figure 1).

\section{DISCUSSION}

Insulin resistance is a pathogenic factor for type 2 diabetes mellitus [17]. Increased insulin secretion and chronic hyperinsulinemia can develop, when pancreatic beta cells can no longer compensate and maintain glucose homeostasis, leading to the development of type 2 diabetes mellitus [18]. In adults, a cut-off value of 2.5 is generally used for HOMA-IR but at present, there is not a wellestablished cut-off for HOMA-IR in children.

One study in obese prepubertal and pubertal

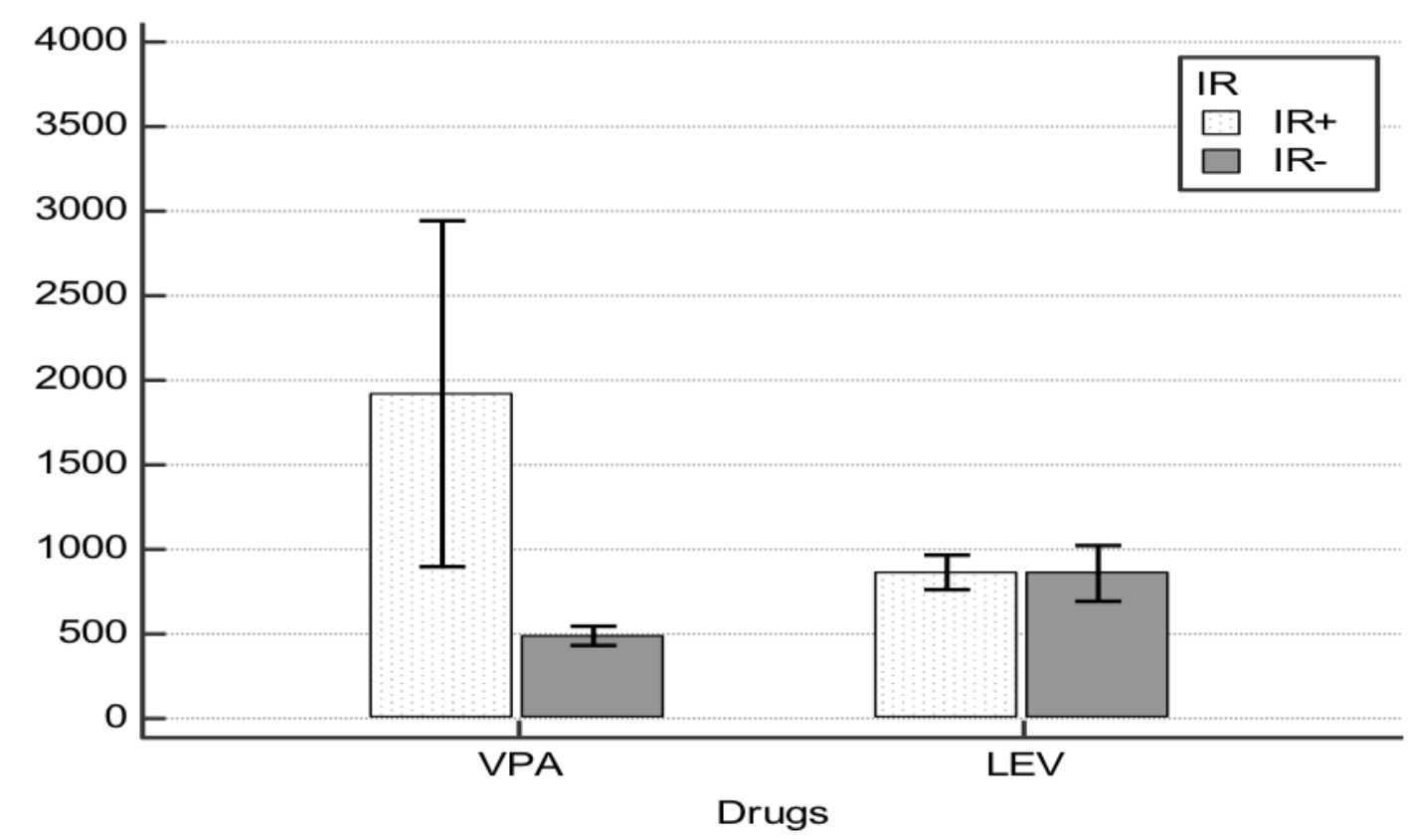

Figure 1. IR positivity increases with increased total dose of VPA. IR (-) = insulin resistance-negative, IR $(+)=$ insulin resistancepositive, $\mathrm{LEV}=$ levetiracetam, $\mathrm{VPA}=$ valproic acid. 
children and adolescents (aged 5-18 years) determined that HOMA-IR cut-off values ranged from 2.67 to 5.22 for boys and 2.22 to 3.82 for girls (19). Another study in 691 apparently healthy Indian adolescents (aged 10-17 years) established a HOMA-IR cut-off of $2.5[20]$.

Notably, in the current study HOMA-IR values were higher than those previously reported in pediatric patients, with an average of 2.6 in normal weight children and 4.1 to 4.3 in overweight and obese children. In a prevalence study, insulin resistance was positive in $28.9 \%$ of females and $25.1 \%$ of males [21]. A pediatric study involving 42 children with a mean age of 10.3 years and normal body weight reported an average blood glucose of $86 \mathrm{mg} / \mathrm{dL}$, an insulin value of $11.9 \mu \mathrm{U} / \mathrm{mL}$ and a HOMA-IR of 2.6 [22]. In our study, mean glucose values were $92.2 \mathrm{mg} / \mathrm{dL}$ and 95.9 $\mathrm{mg} / \mathrm{dL}$ in insulin resistance-positive valproic acid and levetiracetam groups, respectively, which were greater than those found in patients with normal body weight.

In one study, on average, $25 \%$ of overweight individuals had insulin resistance; however, in the present study, $52.5 \%$ of the total study sample tested positive for insulin resistance although our patients had normal body weight [23]. As reported in literature, some children with normal body weight show a higher-than-average HOMA value and the underlying cause of this is not clear. It is possible that children in this age group (9-10 years) have higher HOMA-IR scores, perhaps due to the onset of puberty, where insulin levels have been reported to increase [24, 25]. However, as clearly shown by the current study, antiepileptic drugs have an impact on glucose and metabolism, at least to some extent.

\section{Limitations}

A limitation of the present study is the lack of comparison of data before and after initiation of the antiepileptic medication. Additionally, we did not evaluate other parameters that could influence insulin resistance such as diet, genetic and hormonal factors. Future studies might focus on these and other parameters.

\section{CONCLUSION}

Potential effects of antiepileptic drugs on glucose and insulin metabolism should be investigated in larger patient series and a wider range of antiepileptics. Patients may be screened for insulin resistance before starting antiepileptic treatment and care should be exercised when choosing an antiepileptic drug to avoid potential future development of diabetes mellitus in a susceptible patient. Patients at risk for diabetes mellitus should be educated about measures that help prevent insulin resistance including diet modification and physical activity and antiepileptics associated with development of insulin resistance should be avoided.

\section{Conflict of interest}

The authors disclosed no conflict of interest during the preparation or publication of this manuscript.

\section{Financing}

The authors disclosed that they did not receive any grant during conduction or writing of this study.

\section{REFERENCES}

[1] Edwards HE, Burnham WM, Ng MM, Asa S, MacLusky NJ. Limbic seizures alter reproductive function in the female rat. Epilepsia 1999;40:1370-7.

[2] Edwards HE, Burnham WM, MacLusky NJ. Partial and generalized seizures affect reproductive physiology differentially in the male rat. Epilepsia 1999;40:1490-8.

[3] Feeney DM, Gullotta FP, Gilmore W. Hyposexuality produced by temporal lobe epilepsy in the cat. Epilepsia 1998;39:140-9.

[4] Herzog AG. A relationship between particular reproductive endocrine disorders and the laterality of epileptiform discharges in women with epilepsy. Neurology 1993;43:1907-10.

[5] Banczerowski P, Csaba Z, Csernus V, Gerendai I. Lesion of the amygdala on the right and left side suppresses testosterone secretion but only left-sided intervention decreases serum luteinizing hormone level. J Endocrinol Invest 2003;26:429-34.

[6] Gaitatzis A, Sander JW. The long-term safety of antiepileptic drugs. CNS Drugs 2013;27:435-55.

[7] Jovanovic M, Jocic-Jakubi B, Stevanovic D. Adverse effects of antiepileptic drugs and quality of life in pediatric epilepsy. Neurol India 2015;63:353-9.

[8] Perucca P, Gilliam FG. Adverse effects of antiepileptic drugs. Lancet Neurol 2012;11:792-802.

[9] Shen C, Chen F, Zhang Y, Guo Y, Ding M. Association between use of antiepileptic drugs and fracture risk: a systematic review and metaanalysis. Bone 2014;64:246-53.

[10] Svalheim S, Sveberg L, Mochol M, Tauboll E. Interactions between antiepileptic drugs and hormones. Seizure 2015;28:12-7.

[11] Pennell PB. Hormonal aspects of epilepsy. Neurol Clin 2009;27:941-65.

[12] Harden CL, Nikolov BG, Kandula P, Labar DR, Pannullo S. Effect of levetiracetam on testosterone levels in male patients. Epilepsia 2010;51:2348-51. 
[13] Taubøll E, Gregoraszczuk EL, Tworzydło A, Wójtowicz AK, Ropstad E. Comparison of reproductive effects of levetiracetam and valproate studied in prepubertal porcine ovarian follicular cells. Epilepsia 2006;47:1580-3.

[14] Aygün F, Ekici B, Aydinli N, Aydin BK, Baş F, Tatli B. Thyroid hormones in children on antiepileptic therapy. Int $\mathrm{J}$ Neurosci 2012;122:69-73.

[15] Martin CK, Han H, Anton SD, Greenway FL, Smith SR. Effect of valproic acid on body weight, food intake, physical activity and hormones: results of a randomized controlled trial. J Psychopharmacol 2009; 23:814-25.

[16] Matthews DR, Hosker JP, Rudenski AS, Naylor BA, Treacher DF, Turner RC. Homeostasis model assessment: insulin resistance and betacell function from fasting plasma glucose and insulin concentrations in man. Diabetologia 1985;28:412-9.

[17] Wallace TM, Matthews DR. The assessment of insulin resistance in man. Diabet Med 2002;19:527-34.

[18] Bonora E, Kiechl S, Willeit J, Oberhollenzer F, Egger G, Meigs JB, et al. Population-based incidence rates and risk factors for type 2 diabetes in white individuals: the Bruneck study. Diabetes 2004;53:1782-9.

[19] Gungor N, Hannon T, Libman I, Bacha F, Arslanian S. Type 2 diabetes mellitus in youth: the complete picture to date. Pediatr Clin North Am 2005;52:1579-609.
[20] Kurtoglu S, Hatipoglu N, Mazicioglu M, Kendirici M, Keskin M, Kondolot $\mathrm{M}$. Insulin resistance in obese children and adolescents:HOMA-IR cut-off levels in the prepubertal and pubertal periods. J Clin Res Pediatr Endocrinol 2010;2:100-6.

[21] Singh Y, Garg MK, Tandon N, Marwaha RK. A study of insulin resistance by HOMA-IR and its cut-off value to identify metabolic syndrome in urban Indian adolescents. J Clin Res Pediatr Endocrinol 2013;5:245-51.

[22] Kaya A. Turan E, Uyar M, Bayram F, Turan Y. The prevalence of insulin resistance in the Turkish population: a study conducted with 3331 participants. EJMO 2017;1:202-6.

[23] Beccarelli LM, Scherr RE, Newman JW, Borkowska AG, Gray IJ, Linnell JD, et al. Associations among fatty acids, desaturase and elongase, and insulin resistance in children. J Am Coll Nutr 2018;37:4450 .

[24] Guzzaloni G, Grugni G, Mazzilli G, Moro D, Morabito F. Comparison between beta-cell function and insulin resistance indexes in prepubertal and pubertal obese children. Metabolism 2002;51:10116.

[25] Tobisch B, Blatniczky L, Barkai L. Cardiometabolic risk factors and insulin resistance in obese children and adolescents: relation to puberty. Pediatr Obes 2015;10:37-44. 